\title{
JUSTICIA SOCIOLINGÜÍSTICA PARA LOS TIEMPOS DE HOY
}

\author{
Justiça sociolinguística Para os tempos de hoJe
}

SOCIOLINGUISTIC JUSTICE FOR OUR TIMES

\section{Virginia Zavala}

Magíster y Ph. D. en Sociolingüística, Georgetown University. Profesora

e investigadora, Departamento de Humanidades, sección Lingüística, Pontificia Universidad Católica del Perú, Lima, Perú

Dirección postal: Campus principal, Av. Universitaria 1801, San Miguel, Lima 32, Perú. vzavala@pucp.edu.pe https://orcid. org/0000-0003-3774-5922

\section{RESUMEN}

En este artículo presento la noción de justicia sociolingüística (Bucholtz et al., 2014) en el marco de la tendencia neoliberal de la educación contemporánea, en especial con relación al Perú. En primer lugar, discuto dos significantes de esta tendencia y su vínculo con el fenómeno del lenguaje: la igualdad de oportunidades y la calidad educativa. En segundo lugar, introduzco la noción de justicia sociolingüística y los elementos que la definen, con el objetivo de cuestionar las asunciones del discurso tecnocrático y pensar en políticas del lenguaje más críticas, interculturales y democráticas.

Palabras clave: justicia sociolingüística; lenguaje; igualdad de oportunidades; calidad educativa.

\section{Resumo}

Neste artigo apresento a noção de justiça sociolinguística (Bucholtz et al., 2014) no marco da tendência neoliberal da educação contemporânea, em especial com relação ao Peru. Primeiramente, discuto dois significantes desta tendência e seu vínculo com o fenômeno da linguagem: a igualdade de oportunidades e a qualidade educativa. Em segundo lugar, introduzo a noção de justiça sociolinguística e os elementos que a definem, com o objetivo de questionar as assunções do discurso tecnocrático e pensar em políticas da linguagem mais críticas, interculturais e democráticas.

Palavras-chave: justiça sociolinguística; linguagem; igualdade de oportunidades; qualidade educativa.

\section{Abstract}

In this article, I will present the notion of sociolinguistic justice (Bucholtz et al., 2014) within the frame of the neoliberal tendency in contemporary education, especially in relation to Peru. On the one hand, I will discuss two signifiers of such a tendency and their link with the phenomenon of language: equal opportunities and educational quality for all. On the other hand, I will introduce the notion of sociolinguistic justice and the elements that define it, with the aim of questioning the assumptions of technocratic discourse and delineating more critical, democratic and intercultural language policies.

Keywords: sociolinguistic justice; language; equal opportunities; educational quality.

Recibido: 2018-09-02 / Aceptado: 2019-01-28/ Publicado: 2019-05-20

DoI: $10.17533 /$ udea.ikala.v24n02a09 
Linguistic conventions are quite possibly the last repository of unquestioned authority for educated people in secular society

(Deborah Cameron, 1995, p. 12)

\section{Introducción}

Para nadie es una novedad que la educación está hoy organizada bajo un discurso tecnocrático asociado con las políticas neoliberales. Como lo plantea Stephen Ball (2008), estamos en la era del número, pues existe una reverencia y fijación por el número, la evidencia cuantitativa, la medición, los resultados, la productividad, la performance de los estudiantes y la clasificación de los mismos. Parece ser que, cada vez más, pensamos la escuela como una fábrica generadora de productos estandarizados. Así, nos vamos olvidando de aquellos propósitos sociales de la educación vinculados con la construcción de sociedades más justas e igualitarias (Cuenca, 2012; Torres, 2001). En el Perú, por ejemplo, el objetivo primordial de la educación consiste cada vez más en educar a empresarios y empresarias exitosos que sean suficientemente competitivos para participar en el mercado laboral (y para ser fieles consumidores acríticos) y no en formar ciudadanos críticos, éticos y políticos, como sucedió en la década de 1970 con la influencia de Paulo Freire y la pedagogía crítica (Lamas, 2015; Oliart, 2011). A pesar de que en el Perú también podemos encontrar políticas a favor de la diversidad cultural y lingüística, estas coexisten con medidas educativas neoliberales que las contrarrestan y a veces las acorralan.

En esta oportunidad, me gustaría reflexionar sobre el rol del lenguaje ${ }^{1}$ en los procesos educativos tomando como contexto de fondo la tendencia neoliberal de la educación contemporánea, que se

1 En contraste con la definición más conocida de lenguaje como la facultad biológica exclusivamente humana para adquirir una lengua, aquí utilizo 'lenguaje' como el uso de la lengua en actividades sociales o como las prácticas discursivas que son siempre parte de las prácticas sociales. Utilizo 'lengua' como el conjunto de recursos socioculturales que nos permiten participar en estas actividades comunicativas y sociales. va imprimiendo de diferentes maneras en los países latinoamericanos. En primer lugar, discutiré dos significantes de esta tendencia y su vínculo con el fenómeno del lenguaje: la igualdad de oportunidades y la calidad educativa. En cuanto al primer significante, plantearé que el actual discurso sobre la igualdad de oportunidades puede invisibilizar las desventajas estructurales de grupos minorizados y terminar por reproducir la desigualdad. En cuanto al segundo, señalaré que el discurso sobre la calidad educativa tiende a promover la homogeneización y la reducción de habilidades comunicativas como parte de los procesos estandarizadores. En segundo lugar, y sobre la base de la propuesta de Bucholtz, López, Mojarro, Skapoulli, VanderStouwe y Warner-García (2014), presentaré la noción de justicia sociolingüistica y los elementos que la definen. Esta noción nos servirá para cuestionar las asunciones del discurso tecnocrático y para pensar en políticas del lenguaje más críticas, interculturales y democráticas.

\section{"Igualdad de oportunidades"}

Una primera característica de este discurso tecnocrático se refiere a la valoración instrumental de la educación, como si se tratara de un proceso puramente técnico y por tanto socialmente neutral. Desde esta perspectiva, no se explicitan los intereses y valores sociales que siempre subyacen a las prácticas educativas, y se imponen las políticas sobre la base de lo que los "hechos" (o los resultados de pruebas estandarizadas) demandan (Lemke, 1989). Después de todo, se trata de un discurso que reduce los procesos políticos a mecanismos de gestión, deshistoriza, es competitivo y solo piensa en la rentabilidad a corto plazo.

En el marco de esta valoración instrumental de la educación, se apela a la igualdad de oportunidades, pero muchas veces no se cuestionan las condiciones desiguales en las que se llevan adelante los procesos educativos. De esta manera, se vuelven invisibles los recursos que los estudiantes traen con ellos al punto de partida. Pensemos en muchos de los niños que viven en América Latina. 
La situación de desventaja en la escuela de muchos niños de zonas rurales y de poblaciones indígenas no proviene solamente de la precariedad de la oferta educativa que reciben, sino del hecho de que la socialización vivida durante su primera infancia no coincide con aquella que privilegia y empuja la escuela. Son estas desventajas con relación a lo que el sistema educativo demanda las que se invisibilizan en los argumentos liberales a favor de la igualdad, la justicia y el mérito individual (Augoustinos, Tuffin y Every, 2005; Torres, 2001). Una verdadera política de igualdad de oportunidades funcionará mejor si se contemplan seriamente las discontinuidades lingüísticas y culturales que enfrentan grupos minorizados en la escuela y si nos tomamos en serio el hecho de que lo que se denominan "aprendizajes fundamentales", "lengua estándar", "escritura académica" o "comprensión de lectura" no son comunes a todos los estudiantes y tampoco están disponibles para todos de igual manera.

La sociolingüística, la antropología lingüística y más recientemente la lingüística sociocultural han demostrado que las formas escolares del lenguaje se encuentran presentes en la socialización primaria de niños y niñas de algunos sectores sociales, básicamente de aquellos socioeconómicamente acomodados y con experiencia de vida urbana (Gee, 2005; Heath, 2004; Michaels, 1988, entre muchos otros). Vale decir que muchos niños crecen en un ambiente en el que las formas de conocer, de aprender y de interactuar son las típicamente escolares. Aprender a nombrar objetos desde muy pequeños, a contestar preguntas cuyas respuestas ya conoce el interlocutor, a narrar oralmente con un formato típico del texto escrito de corte expositivo, a utilizar los recursos lingüísticos de forma descontextualizada y explícita, a realizar performances orales para mostrar sus habilidades a un público adulto y esperar recompensa constituyen solo algunas de las prácticas con el lenguaje que se encuentran en el centro de la socialización primaria de muchos niños y niñas, quienes al ingresar a la escuela perciben una continuidad con lo que recibieron en sus hogares. Otros niños y niñas, en cambio, se socializan de maneras muy diferentes a las formas escolares: aprenden diferentes nociones y habilidades a través de la observación, la imitación y la práctica, y no centran su aprendizaje en la comunicación verbal (Philips, 2001; Scollon y Scollon, 1981). Así entonces, como lo han demostrado los estudios dentro del campo de la socialización lingüística (De León, 2010), muchos niños no solo pueden traer a la escuela una nueva lengua (en el caso de aquellos que hablan una lengua originaria), o incluso una variedad gramatical distinta de la estándar, sino también maneras culturales de usar la lengua y valores e ideas con relación a la enseñanza y el aprendizaje de la misma que difieren de la cultura escolarizada hegemónica. Y es que la lengua no es un objeto aislado que se aprende técnicamente. El uso de la lengua siempre es parte de lo que hacemos socialmente, de cómo nos asumimos a nosotros mismos y a los otros, y de lo que creemos y valoramos como parte de nuestro lugar en el mundo (Gee, 2005).

No obstante, todo esto está invisibilizado en las políticas educativas en el Perú. El discurso del constructivismo que se expandió en la década de 1990 (y los conceptos de saberes previos, aprendizajes significativos o metodología activa) no ha considerado el tema cultural a cabalidad ni ha permitido combatir la discriminación pedagógica en el aula (Ruiz Bravo, Rosales y Neira, 2006). La prueba PISA también cuenta con una noción estrecha de cultura a la hora de reconocer factores asociados al rendimiento de los niños. Cuando hace referencia a los aspectos comunicativos, puntualiza la "posesión de bienes de carácter cultural (literatura clásica, libros de poesía y trabajos de arte)" y el "diálogo que se desarrolla entre padres e hijos en torno a temas particularmente referidos a aspectos culturales".2 Sorprende una noción de cultura entendida como una práctica reguladora de un gusto autodenominado "universal" que se nombra como la única expresión cultural. Pero lo

2 Ver reportes sobre factores asociados en su página web. 
importante no solo es revelar que la prueba PISA habla sobre "lo culto" definido desde la hegemonía, sino también darnos cuenta de que invisibiliza todo lo que acabo de señalar como un factor que de hecho está asociado al rendimiento. A pesar de que se enfatizan las temáticas de interacción, se silencian las maneras de interactuar con el lenguaje, maneras que luego colocarán a muchos niños en clara desventaja con relación a otros en el proceso escolar.

El discurso de la igualdad de oportunidades no puede funcionar bajo la ilusión de que todos somos iguales y de que las desventajas solo existen a nivel de logros individuales y pueden superarse con esfuerzo y determinación. Este discurso debe tomar en cuenta las desventajas estructurales de ciertos sectores de la sociedad con relación a la escuela y además visibilizar el rol que juega el lenguaje en estas desventajas.

\section{6}

\section{"Calidad educativa"}

Una segunda característica del discurso tecnocrático es la homogenización (y la reducción) de las habilidades que se buscan impartir a los estudiantes. A la obsesión por las pruebas estandarizadas subyace una asociación entre la aplicación de las pruebas y la evaluación de la calidad de la educación, pues se asume que una buena educación es aquella que constata la adquisición de los conocimientos medidos en las pruebas (Luke, Green y Kelly, 2010). Además de esto, se asume que las habilidades que se evalúan en las pruebas son universales, objetivas y apolíticas, cuando sabemos que estas no son habilidades objetivas, sino habilidades particulares que se relacionan con prácticas específicas y situadas. Aunque se asume que la información supuestamente "objetiva" recogida en este tipo de pruebas ayudará a los educadores a detectar problemas educativos, sabemos que los resultados cuantitativos no cuentan la historia completa de la problemática educativa, sino que solo muestran lo que los niños pueden o no pueden hacer en relación con las habilidades que miden las pruebas. Los resultados de las pruebas no revelan necesariamente aprendizajes auténticos y contextualizados, sino la forma en que los estudiantes han prestado atención al formato de la prueba y han practicado las reglas de juego de las convenciones implicadas, de una forma mecánica y desde una pedagogía descontextualizada (Zavala, 2012).

Al igual como ha sucedido en otros contextos, en el Perú, la manera como se han manejado los resultados obtenidos en las pruebas ha tenido consecuencias en el trabajo en el aula y en la reducción de habilidades que se busca impartir a los estudiantes (Au, 2011; Amrein y Berliner, 2002). La enseñanza para la prueba empobrece el currículo, pues las áreas que no son consideradas en ella comienzan a recibir menos atención. La lectura también adquiere más prominencia frente a las destrezas orales, que poco a poco se dejan de desarrollar en los educandos. En relación con la lectura, esta empieza a asociarse solo con las prácticas que requieren las pruebas estandarizadas de comprensión lectora. Así, por ejemplo, los maestros suelen medir la comprensión de sus estudiantes solamente utilizando preguntas cerradas que buscan información breve y tratando de confirmar que estos conocen las respuestas verdaderas. Los docentes no hacen comentarios ni proveen información que trasciende el ejercicio tipo prueba y su participación está más enfocada en el formato de los ejercicios que en el contenido académico (Zavala, 2012). De esta manera, estas prácticas de lectura limitan la vasta gama de habilidades que los estudiantes podrían desarrollar en la escuela y los posicionan como receptores pasivos de información, sin agencia ni creatividad, y que solo participan en las actividades para demostrar su destreza en las pruebas. Peor aún, los estudios han demostrado que los alumnos que hablan lenguas originarias y que van a escuelas rurales son más proclives a recibir un tipo de instrucción que se enfoca en la preparación para la prueba en la forma de ejercicios repetitivos de memorización, debido a que es precisamente esta población la que no obtiene buenos resultados en las pruebas estandarizadas (Cummins, 2007; Madaus y Clarke, 2001; 
Piller, 2016). Esa especial atención y preparación para las pruebas ocurre en desmedro de otros tipos de oportunidades de aprendizaje y otros métodos de enseñanza que podrían ser más efectivos para las necesidades de esta población estudiantil (Menken, 2010).

Ahora bien, esta reducción de habilidades en los estudiantes está ocurriendo en un mundo donde - paradójicamente- se necesitan muchas más habilidades que antes. La multiplicidad de canales de comunicación, la globalización, la creciente urbanización, los procesos de migración y los mayores contactos y flujos entre los grupos sociales no solo requieren otro tipo de aprendizajes, sino que incluso modifican la naturaleza misma de lo que significa aprender y de las identidades que se proyectan en el mundo. Todo esto requiere una mirada más amplia de la comunicación y de la literacidad que lo que se implementa con enfoques lingüísticos tradicionales centrados en la "corrección" y en el dominio individual de un conjunto de reglas (Gee, 2005). Es más, impartir solo el español académico a los estudiantes puede abrirles ciertas puertas, pero también limitar su repertorio comunicativo y los tipos de encuentros que podrían tener en diferentes contextos. Con razón argumenta Gee (2005) que la literacidad académica es una condición necesaria, pero no suficiente para participar en el mundo de hoy y tener "éxito en la sociedad", pues es parte de lo que él llama las "viejas literacidades". Si bien el lenguaje académico se consideró alguna vez como central para poder hablar de una persona "escolarizada" e "inteligente", hoy en día su adquisición debe ir unida a otras cosas (como a las habilidades para hacer búsquedas en internet, distinguir información confiable de la que no lo es, o producir discursos multimodales).

\section{Una educación con justicia sociolingüística}

Desde sus inicios, la sociolingüística se constituyó en un proyecto tanto intelectual como político interesado por la justicia social. Más aún, a lo largo de las décadas este campo — cada vez más interdisciplinario - se ha hecho preguntas con relación a cómo el lenguaje impacta en las tres dimensiones que, según Fraser (2005), son fundamentales para pensar en la justicia social: la redistribución económica, el reconocimiento cultural y la participación política (ver Piller, 2016, para una discusión más actual). Recientemente, la categoría de 'justicia lingüística' ha sido discutida a partir de perspectivas económicas y filosóficas en relación al impacto de las políticas lingüísticas (Gazzola, Templiny Wickström, 2018).Conciente de la pluralidad de enfoques para abordar el tema, en este trabajo echaré mano de la noción de 'justicia sociolingüística' propuesta por Bucholtz et al. (2014) para hacer referencia a una educación lingüística que empodere a los individuos y grupos subordinados en batallas sociopolíticas en torno al lenguaje. Si bien esta noción se circunscribe más al ámbito de la educación y la escuela, parto de la idea de que las desventajas lingüísticas deben ser asumidas como desventajas estructurales (Piller, 2016). En el marco de esta noción de justicia sociolingüística, los autores discuten una serie de objetivos ${ }^{3}$ que presentaré a continuación con ejemplos de mis propias investigaciones y de otros estudios.

\section{Valoración}

Este primer objetivo se refiere a promover conciencia y apreciación de la variación y la diversidad lingüística de todos los tipos, y a comprender la sistematicidad, complejidad y valor cultural de las formas y prácticas lingüísticas tanto de uno mismo

3 En este trabajo no reproduzco los cinco objetivos que presentan Bucholtz et al. (2014), sino que los reinterpreto y los inserto en una nueva clasificación que se reduce a tres (valoración, legitimación y acceso). Por un lado, he insertado el objetivo tres sobre 'herencia lingüística' (que los autores mantienen como un objetivo separado) en el objetivo uno de 'valoración'. Por otro lado, no he desarrollado el objetivo cinco en torno a la experticia lingüística. Este objetivo se refiere a la necesidad de reconocer que todos los usuarios de lenguas son expertos en la temática y deberían participar más activamente en la producción de conocimiento académico. 
como de los otros (Bucholtz et al., 2014). Esto implica la conciencia y apreciación sobre diferentes formas asociadas a lenguas y variedades a nivel gramatical y abstracto, ${ }^{4}$ pero también sobre diferentes prácticas lingüísticas en diferentes tipos de contextos. Aquí se incluyen, por ejemplo, las formas orales de socialización con el lenguaje que aprenden muchos niños de diversos contextos culturales y que entran en conflicto con la cultura escolar (De León, 2010), así como muchas prácticas vernáculas de leer y escribir que tampoco se alinean con las maneras de leer y escribir de la escuela, pero que cumplen propósitos importantes en la vida de las personas y las comunidades (Zavala et al., 2004).

Me gustaría empezar llamando la atención sobre lo que los maestros suelen enseñar cuando se hace referencia a la lengua. Ya desde hace varios años los enfoques sociolingüísticos han pasado de analizar la lengua como un sistema abstracto al lenguaje como práctica y como usos situados en contextos específicos y en el marco de propósitos particulares. Esto último nos lleva a mirar la lengua desde la noción de repertorio comunicativo, entendido como el conjunto de recursos que tienen los individuos para funcionar efectivamente en las múltiples comunidades en las que participan (Rymes, 2014). Un repertorio puede incluir múltiples "lenguas", "variedades" y formas de hablar, pero también gestos, formas de vestir, posturas, rutinas comunicativas y patrones de entonación reconocibles. La lengua - en términos de recursos gramaticales y léxicos- sería solo una parte de un repertorio comunicativo, pues siempre se usa en el marco de acciones y actividades situadas, y del despliegue de una diversidad de posicionamientos identitarios. Es precisamente este uso de elementos del repertorio en contextos específicos

4 Soy conciente de que las lenguas son constructos culturales y no realidades objetivas. Por esta razón, a veces coloco el término entre comillas o hago referencia a las formas asociadas a lenguas históricamente identificables. En lugar de hablar de 'lenguas' prefiero hablar de 'recursos lingüísticos'. (aquello que estoy denominando 'lenguaje') lo que termina generando la asociación entre recursos lingüísticos y significados sociales particulares.

El desarrollo individual a lo largo de la vida consiste - en parte- en la creciente acumulación de recursos comunicativos y en la toma de conciencia sobre sus significados sociales (o lo que estos indexan) en diferentes situaciones. Es importante que los estudiantes sean concientes de su propio repertorio comunicativo y de sus efectos en diversos contextos. Esta toma de conciencia puede realizarse, por ejemplo, con relación a las diferentes formas de usar nombres, apodos y fórmulas de tratamiento, referencias a la cultura popular, gestos, patrones de alternancia de turnos, narraciones, formas asociadas a diferentes "lenguas" en una misma situación comunicativa, saludos, chistes, tipos de pronunciación, arreglos para integrar lo verbal con otros modos comunicativos (como fotos, emoticones y música), etc. Uno de los objetivos de la escuela sería que los estudiantes expandan y enriquezcan su repertorio comunicativo para vivir con otros (Rymes, 2014).

No obstante, si bien los repertorios de los estudiantes deben acomodarse a las rutinas y repertorios escolares, la acomodación tendría que ser bidireccional, en el sentido de que los docentes también deberían acomodarse a los repertorios que traen los estudiantes de su socialización primaria y aquellos que van aprendiendo fuera de la escuela como parte de su contacto con diferentes comunidades de práctica. Esto implica dejar de enfocarnos solamente en la forma correcta y "apropiada" del lenguaje e intentar movernos a través de las fronteras discursivas, para que así la conexión humana y el aprendizaje relevante puedan ocurrir (Rymes, 2014). Si los docentes aprenden que los repertorios de los estudiantes son valiosos, estos comienzan a verse a sí mismos como más capaces académicamente, en el sentido de más capaces también de expandir su propio repertorio y de construir algo nuevo sobre la base de él. Por lo tanto, la conciencia en torno a la diversidad de repertorios comunicativos no sería un medio para llegar al 
uso "correcto" del lenguaje, sino un fin en sí mismo, que sirve a los estudiantes para darse cuenta de que deben contar con un conjunto de recursos comunicativos para desarrollar un sentido de pertenencia compartida en más comunidades.

Este primer objetivo también incluye el derecho que tienen las personas a valorar, tomar conciencia y aprender (o reaprender) una "lengua de herencia” que se ha perdido o está en vías de desaparición y que remite a la historia y experiencias de los estudiantes (Bale, 2010; Leeman y King, 2013; Van Deusen-Scholl, 2003). Lo importante de estos estudiantes - o de estos hablantes de una "lengua de herencia" - es que a pesar de que pueden no manejar la lengua involucrada con fluidez (o quizás ni siquiera comprenderla) mantienen vínculos familiares, étnicos o identitarios con la lengua y la cultura en cuestión, y han crecido en hogares donde la lengua ha estado presente de una forma u otra en la comunicación familiar. Este es el caso de muchísimos niños y niñas que viven en nuestro continente. A pesar de que la oficialidad generalmente los identifica como hablantes de la lengua originaria como segunda lengua y sin derecho a aprenderla en el proceso escolar, muchos estudiantes "la entienden pero no la hablan" y se sienten conectados a ella culturalmente. La escuela debería socializar a estas nuevas generaciones en las tradiciones lingüísticas y culturales de generaciones previas.

Los estudiantes pueden "reconectarse" con esta lengua, aunque esto no implique dominarla totalmente, sino echar mano de ella de manera selectiva. De hecho, aunque los educandos solo desarrollen un compromiso parcial con una lengua o variedad que se asume como parte de la herencia, esta reconexión puede impactar poderosamente en su identidad lingüística y cultural. Después de todo, el objetivo es reforzar el sentido de conexión del estudiante con el pasado lingüístico de su familia, pero también con un futuro donde esta lengua esté presente y se desarrolle, sobre todo en el marco de una escuela que históricamente ha funcionado desarraigando a los educandos de sus contextos culturales (Zavala, 2015).

Ahora bien, más allá de promover conciencia sobre la variación, es importante que los estudiantes la aprecien y la valoren. Esto, sin embargo, no puede hacerse desde un discurso multiculturalista celebratorio que invisibiliza el poder (Žižek, 1997), sino en el marco de una discusión sobre las ideologías lingüísticas que se forman a partir del uso, pero que también lo gobiernan. Después de todo, la diversidad siempre está jerarquizada. Esto nos lleva al segundo objetivo propuesto por Bucholtz et al. (2014).

\section{Legitimación}

Los encuentros cotidianos entre las personas están siempre atravesados por relaciones de poder, que influyen sobre la posibilidad del uso de ciertos recursos en contextos comunicativos particulares. Como lo plantean Blommaert y Backus (2011), mientras que algunos recursos (como los vinculados con las variedades prestigiosas de una lengua) pueden circular a través de un rango amplio de situaciones, otros (como aquellos vinculados con el uso del quechua en el Perú, por ejemplo) tienen un potencial de movilidad muy restringido. En ese sentido, resulta clave analizar el valor indexical o las connotaciones sociales que se han construido sobre diferentes tipos de recursos lingüísticos en ciertos espacios y situaciones.

Este segundo objetivo busca promover la validez de los repertorios lingüísticos de los estudiantes, para el uso comunicativo y simbólico, en un amplio rango de esferas sociales, incluyendo no solo los espacios íntimos del hogar y la comunidad, sino también aquellos más formales, públicos e institucionales. Este segundo objetivo va más allá de la valoración, e incluye dos aspectos por trabajar: 1) la promoción de una conciencia lingüística crítica en el sentido de "una discusión explícita en torno a los asuntos del poder y la ideología que subyacen a las prácticas del lenguaje" (Clark e Ivanič, 1997), y 2) la acción misma para transformar aquellas representaciones en torno al lenguaje 
que sustentan formas de dominio. Vale decir que la concientización en términos explícitos se refuerza cuando los docentes también se comprometen con la práctica "oposicional” y con sus acciones desafían lo que se proyecta como el orden sociolingüístico "natural" (Zavala, 2015). Comenzaré por el primer aspecto y luego continuaré con el segundo.

Como señalé anteriormente, los recursos lingüísticos adquieren valores indexicales que los conectan ideológicamente con otros fenómenos sociales y con las personas que los despliegan. Es de esta manera como se construyen las ideologías lingüísticas o las valoraciones sobre el lenguaje, que siempre camuflan ideologías de otro tipo y que están estrechamente vinculadas con el poder (Irvine, 1989; Kroskrity, 2000; Woolard, 1998; Rosa y Burdick, 2017). Así, por ejemplo, la ideología sobre el motoseo (o sobre la interferencia vocálica del quechua en el castellano de los bilingües en el Perú) conlleva una representación en la que el fenómeno constituye un error o un defecto de un sujeto que es concebido como inferior a aquel que no tiene interferencias. Específicamente, el sujeto motoso es representado como un indio de alguna comunidad campesina de altura, poco inteligente e incapaz de opinar legítimamente sobre el devenir social (Zavala y Córdova, 2010). Aunque la interferencia vocálica no necesariamente refleja las características mencionadas, el poder de la ideología se encarga de unir el fenómeno lingüístico con un determinado grupo social - y una serie de características peyorativas - en un vínculo que termina percibiéndose como natural y verdadero. De esta manera, se termina culpando a los sujetos por ser motosos y no se cuestiona la forma en que la ideología ha racializado el rasgo lingüístico para legitimar la discriminación. Después de todo, las ideologías lingüísticas siempre se conectan con ideologías sobre los hablantes involucrados y sobre otros aspectos de la estructura social.

Del mismo modo, podríamos generar una reflexión sobre la ideología de la lengua estándar (Milroy, 2007; Siegel, 2006). La lengua estándar constituye una forma abstracta e idealizada de lengua que se basa en el habla de los estratos sociales privilegiados y que la escuela se encarga de enseñar, aunque no siempre con éxito. Se trata entonces de una variedad imaginada, pero que sí se asocia con cierto tipo de rasgos y prácticas lingüísticas. $\mathrm{Al}$ igual que con el caso del motoseo, la ideología se ha encargado de construir una representación de la lengua estándar que se asume como verdadera y que reproduce la desigualdad. ¿Qué representación es la que se asume como verdadera? Se asume que esta variedad es intrínsecamente superior a cualquier otra, que ha sido elegida por consenso social y de forma democrática, que todos tenemos las mismas oportunidades de aprenderla si es que nos esforzamos lo suficiente y que ya no seremos discriminados y ascenderemos socialmente si decidimos usarla correctamente en los contextos donde es apropiado hacerlo.

Si deconstruimos la ideología, en primer lugar podemos darnos cuenta de que la variedad estándar (o lo que nos imaginamos que es) no es intrínsecamente superior a otras variedades, sino que ha sido construida socialmente como superior a partir de relaciones de poder. Si otra hubiera sido la historia y otros sectores sociales hubieran estado en el poder, probablemente concebiríamos a otra variedad como la ideal o el modelo a seguir. En segundo lugar, tampoco se trata de una variedad que ha sido elegida por consenso y de forma democrática. Ni siquiera es una variedad que hable la mayoría de la población. Los propios lingüistas han construido una representación ideológica en torno al estándar como aquella variedad socialmente aceptada, como la más adecuada para los contextos formales de uso, como la forma socialmente preferida, y como aquella que ha surgido a partir de un acuerdo social más o menos tácito con el fin de entendernos perfectamente entre todos. Es más, incluso se ha planteado que son los hablantes los que deciden democráticamente qué es estándar y qué no, y qué formas de habla son adecuadas para qué tipo de contexto (Bernárdez, 2004). No es difícil darnos cuenta, sin embargo, de que son los sectores privilegiados los que manejan la variedad 
estándar, que la gran mayoría no la conoce, que esta no necesariamente nos permite entendernos entre todos, que constituye la variedad ideal precisamente porque es la que representa a los sectores de élite y que estos últimos - y no todos- son los que deciden qué es estándar o no y qué forma es la apropiada para los diferentes contextos. La ideología construye la ilusión de un acuerdo en torno al estándar para negar los antagonismos y seguir sustentando formas de dominio a través del consenso y no de relaciones coercitivas o medidas a la fuerza. Las relaciones de poder han generado una "verdad" en torno a la variedad estándar, una verdad que ya es parte del sentido común y que es justificada y defendida por los propios actores que resultan perjudicados por ella. Se trata de un claro ejemplo de la premisa ideológica que presenta el interés propio como el interés común y general de todos los miembros de la sociedad (Marx y Engels, 1968, p. 53).

En tercer lugar, sabemos que no todas las personas están en la misma posición con relación al aprendizaje de esta variedad. Hay niños y niñas que han aprendido una variedad cercana al estándar como parte de su socialización primaria, y hay otros que han aprendido una variedad muy lejana al estándar y a los que les cuesta más aprenderla en la escuela, pues ya han adquirido una variedad diferente. No todos los niños tienen el mismo bagaje lingüístico al inicio de la etapa escolar y, si los maestros no son concientes de esto, la brecha entre el lenguaje que traen de la casa y el lenguaje que la escuela impone puede agrandarse aún más. Aunque el esfuerzo puede ser un componente importante, no debemos centrar la problemática en el sujeto, tal como la ideología lo hace, pues se trata de desventajas estructurales que no se suelen visibilizar.

En cuarto lugar, por más que la persona aprenda la variedad estándar, puede seguir siendo discriminada a partir de otros criterios, como los raciales, por ejemplo, pues las maneras de usar la lengua siempre son parte (y no pueden separarse) de otros signos y conductas que adquieren carga ideológica. Lo anterior se vincula con una idea muy potente propuesta por Flores y Rosa (2015) con relación a la importancia de considerar no solo la producción lingüística del enunciante, sino también la percepción del oyente. Esto implica, a su vez, concebir el uso de la lengua como parte de prácticas sociales particulares y siempre en el marco de interacciones con otros que posicionan a los hablantes desde categorías específicas. Estos autores lo explican claramente: los interlocutores no perciben la forma lingüística del enunciante aislada de su contexto, sino siempre en el marco de su posicionamiento identitario y de categorías vinculadas con su clase social, su procedencia, su fenotipo, sus prácticas culturales, entre otras. Las prácticas del lenguaje están siempre unidas con otras prácticas sociales y no pueden disociarse de estas últimas en el imaginario de las personas, en contraposición con lo que ocurre con el trabajo de muchos lingüistas, que se focaliza en el sistema lingüístico, aislado de las prácticas sociales.

A partir de lo anterior, se deduce que si una persona produce las prácticas lingüísticas estándares, el oyente nunca las percibe como conjuntos objetivos de formas lingüísticas, sino que las escucha como parte de un posicionamiento particular en el mundo social y muchas veces de un cuerpo racializado. En ese sentido, el oyente puede atribuir "deficiencia lingüística" al hablante sobre la base de su posición racializada en la sociedad y no tanto de un rasgo lingüístico objetivo. Por lo tanto, a pesar de que el hablante es capaz de cambiar su forma de hablar, esto no siempre modifica su posicionamiento social desde las perspectivas ideológicas de los sujetos que escuchan. Desde esta mirada, entonces, la discriminación lingüística debe ser entendida no tanto como un reflejo de prácticas lingüísticas objetivas, sino como percepciones construidas sobre la base de las posiciones de los hablantes. Como consecuencia, los individuos no pueden controlar las formas en que su forma de hablar es interpretada por sus interlocutores.

En esta misma línea, Blommaert, Collins y Slembrouck (2005) hacen referencia a que el uso 
de las lenguas (o de los recursos lingüísticos en general) no depende tanto de la capacidad del individuo, sino de un entorno que lo inhibe, constriñe, discrimina o inferioriza. En ese sentido, las personas con habilidades multilingües desarrolladas pueden sentirse incapacitadas comunicativamente cuando se encuentran "fuera de lugar”. Un cambio en el ámbito espacial (como, por ejemplo, un cambio del ámbito privado al ámbito público o del campo a la ciudad, en el caso del quechua) claramente afecta la capacidad de los usuarios para desplegar recursos lingüísticos y habilidades, e impone requerimientos en ellos. Por lo tanto, no estamos hablando de un problema del hablante, sino de un problema para el hablante, producido por los requerimientos del ámbito, en el sentido de que la función y el valor de los recursos en ese ámbito en particular adquieren otros significados. Es el espacio social (y las relaciones de poder inscritas en él) el que condiciona, en gran medida, la organización de patrones del lenguaje, sobre todo en el caso de recursos con un potencial de movilidad restringido.

Pondré un ejemplo para ilustrar lo anterior. En el Perú, muchos quechuahablantes suelen hablar en quechua en las zonas rurales, pero en castellano cuando van a las ciudades. Esta constatación no puede llevarnos a argumentar que así son las cosas y que lo apropiado consiste en separar las lenguas de acuerdo a estos contextos geográficos. Por el contrario, deberíamos analizar cómo este estado de cosas ha llegado a constituirse como tal debido a relaciones de poder. En efecto, en las ciudades del Perú los quechuahablantes suelen hablar en castellano, pues hablar en quechua implica ser visto como una persona que no ha aprendido el castellano y, por ende, como pobre, carente, con bajo nivel de escolaridad y proveniente de una comunidad campesina de altura. En otras palabras, hablar en quechua en la ciudad equivaldría a una especie de confesión de inferioridad con relación al interlocutor, pues implica proyectar una imagen vulnerable que posiciona al hablante como carente (Zavala et al., 2014). Con este ejemplo, podemos ver que el quechuahablante es silenciado en su lengua originaria por un oyente que lo posiciona como inferior cada vez que la habla. Pero también podríamos subrayar lo que Flores y Rosa (2015) plantean: que el uso de formas asociadas al castellano en lugar de al quechua no necesariamente modifica el posicionamiento racializado del hablante en el imaginario del oyente. A pesar de hablar en castellano y reprimir el quechua, probablemente el hablante siga siendo percibido como indio, con la carga peyorativa que esto trae consigo.

A partir de lo anterior, podemos cuestionar la ideología de la igualdad de oportunidades asociada a la lengua estándar, según la cual si los sujetos modifican sus prácticas lingüísticas dejarán de ser discriminados y se eliminarán las jerarquías sociales. Y es que no se logra la erradicación de la discriminación lingüística solo estableciendo la legitimidad de todas las prácticas lingüísticas. Más allá de analizar las formas lingüísticas de forma descontextualizada, es importante abordarlas como parte de posiciones de enunciación y de recepción (Flores y Rosa, 2015). Solo con esta perspectiva podríamos darnos cuenta, por ejemplo, de que dos personas que emiten el mismo rasgo (el motoseo, por ejemplo) pero desde dos posicionamientos sociales diferentes pueden provocar reacciones distintas por parte de los interlocutores (de aceptación en un caso y de rechazo en otro), o que dos personas que emiten rasgos distintos pueden provocar la misma reacción (de rechazo en ambos casos) por parte de los oyentes. Así, entonces, la enseñanza de formas lingüísticas escolares a poblaciones vulnerables debe ir más allá de enseñar reglas de "lo apropiado", con el objetivo de abordar conflictos y batallas más amplias que ofrezcan a estos estudiantes herramientas para desafiar las inequidades que enfrentan. El empoderamiento no se logra solo dándoles acceso a las formas lingüísticas del poder, sino formándolos para que se conviertan en agentes transformadores del poder.

La segunda parte de este objetivo se vincula con la posibilidad de que los maestros puedan 
transformar estas ideologías con intervenciones en la práctica. Hemos visto que lo central en este segundo objetivo es el tema del poder. Sin embargo, concuerdo con Street (1996) cuando sostiene que para los educadores preocupados por el tema del poder, la pregunta no es cómo unos pocos pueden acceder a él o cómo las estructuras de poder pueden ser resistidas, sino cómo el poder puede ser transformado. Según esta mirada, entonces, debemos preguntarnos cómo los docentes pueden contribuir a la transformación del poder, en el sentido de desafiar y desestabilizar representaciones y relaciones sociales dominantes que han sido históricamente reproducidas en la escuela.

Lo que hay que discutir, entonces, es que el uso de los recursos lingüísticos siempre está atravesado por el poder. Precisamente es el poder lo que hace que algo se diga de esa manera (y no de otra) en alguna circunstancia o, como lo plantea Kress (2001), lo que finalmente explica la variación en el lenguaje. Ahora bien, esto no significa asumir una perspectiva de "lo apropiado" que ha desarrollado la sociolingüística de corte más "liberal" en las últimas décadas con relación a la variedad estándar y a la literacidad académica (Pennycook, 2001; Fairclough, 1995; Flores y Rosa, 2015). Desde este paradigma, la idea es enseñar las formas "apropiadas" en los momentos "apropiados" desde una perspectiva normativa que asume las convenciones dominantes de lo apropiado como naturales y necesarias. En esta línea, se sugiere que los estudiantes mantengan sus discursos del hogar en el hogar y que ingresen al discurso académico con una nueva identidad y sentido de la realidad. Una sociolingüística de este tipo no hace sino reproducir los arreglos ideológicos dominantes, pero con un nuevo discurso a favor del "respeto" por los estudiantes. Como bien lo ha anotado Leeman (2005), decirles a los estudiantes que sus variedades lingüísticas son correctas, pero a su vez inapropiadas para el contexto académico y profesional naturaliza el tratamiento desigual de las variedades lingüísticas y de sus hablantes, y disfraza la prescripción lingüística como descripción inocente. Durante años los lingüistas hemos señalado lo anterior, sin darnos cuenta de que es lingüísticamente correcto, pero socialmente muy ingenuo.

Este aspecto de la legitimación implica precisamente intervenir para transformar las representaciones y prácticas en torno al lenguaje que reproducen la desigualdad y desafiar estas asociaciones entre maneras de hablar y posicionamientos sociales particulares. Presentaré algunos ejemplos que muestran cómo se puede contribuir a las transformaciones sociales desde los espacios educativos, aunque sabemos que lo que ocurre dentro de la escuela debe reforzarse con cambios en otros espacios. Los primeros ejemplos provienen del contexto norteamericano. En Estados Unidos los estudiantes latinos bilingües son estimulados a usar sus habilidades en español cuando se les pide que recolecten datos lingüísticos y que luego presenten sus resultados de investigación tanto en inglés como en español. De esta manera, las familias y otros miembros de la comunidad pueden darse cuenta de que el uso del español fortalece, no debilita el desarrollo académico de los estudiantes. Además, estos pueden experimentar el español como una lengua de considerable valor institucional, en el marco de representaciones dominantes en las que se asume lo contrario (Velasco y García, 2014). Así también, la investigación ha recogido diversas iniciativas con relación a pluralizar las convenciones de la escritura académica con los estudiantes en las aulas. No solo se han incorporado literacidades vernáculas, y específicamente digitales, para el trabajo escolar, sino que se han promovido formas asociadas a dos lenguas (o dos variedades), o diversas estrategias discursivas en la escritura académica, con el objetivo de pluralizar este tipo de escritura y estimular la inserción de la voz de los estudiantes (Canagarajah, 1997).

También puedo presentar ejemplos que provienen de los Andes peruanos. En este contexto, un maestro en una universidad local se esfuerza por elogiar a los estudiantes "motosos" (o a aquellos que tienen 
interferencias fonológicas del quechua en su castellano) cuando exponen en las aulas y, de esta manera, trata de deconstruir la representación que asocia la variedad motosa con poca inteligencia, poca coherencia en la expresión de ideas y pocas probabilidades para rendir bien en la escolaridad (Zavala y Córdova, 2010). Así, en lugar de avalar la burla de los demás compañeros hacia estos estudiantes, intenta posicionar a estos últimos de formas no esperables y contrarrestar la ideología dominante. En el mismo contexto, pero desde la educación primaria, otra maestra les da visibilidad al quechua y a quienes tienen usos más fluidos de la lengua en la escuela urbana donde trabaja. De esta manera, desafía las prácticas "esperables" en este tipo de institución, donde el quechua se margina y los estudiantes de procedencia más rural no suelen tener voz en el salón. Al darles voz a estos estudiantes y legitimar su participación en quechua, los posiciona como expertos en esta lengua y subvierte una visión paternalista y tutelar que se ha construido históricamente hacia ellos (De los Heros, 2012; Zavala, 2015). Se podrían poner muchos más ejemplos. De lo que se trata, en todo caso, es de ser crítico con el estado de cosas que se naturaliza y de intentar desestabilizarlo a partir de las prácticas escolares.

La idea es dejar atrás visiones positivistas en torno a las cosas como son y pasar a una perspectiva posestructuralista sobre cómo el estado de cosas ha llegado a ser lo que es y cómo se pueden modificar situaciones de desigualdad e injusticia. Desde esta mirada, es importante preguntarnos si una política educativa que promueva la igualdad de oportunidades y el empoderamiento de los sujetos significa solamente enseñar los recursos lingüísticos asociados al poder o, también, desafiar las normas que designan a un conjunto limitado de recursos como las claves para la movilidad y el cambio social.

\section{Acceso}

Este tercer objetivo se refiere a la necesidad de enseñar (y de aprender) las lenguas, variedades y formas de hablar legitimadas por el poder.
La valoración y legitimación de variedades marginadas de ninguna manera sustituye el acceso a las lenguas y maneras de hablar dominantes por parte de todos los estudiantes. Todos sabemos que la escuela requiere que los estudiantes aprendan a usar las lenguas de nuevas maneras, tanto en nuevas formas de interacción como en la producción de nuevos tipos de textos (Schleppegrell, 2005). No obstante, a pesar de que el proceso escolar supuestamente se centra en promover el dominio de estas formas y de que los estudiantes están expuestos a las variedades dominantes a través de la educación, muchos pueden seguir teniendo dificultades para acceder a ellas debido a prácticas educativas que no construyen sobre las maneras de abordar el lenguaje que los estudiantes traen de sus hogares. Más aún, muchos docentes no logran enseñar a los niños estas variedades académicas de lenguaje, porque no son concientes de que existen, de las convenciones que subyacen a ellas y de que tienen que ser aprendidas por los educandos (Snow y Uccelli, 2009). Como lo plantea Gee (2005), por lo general, a los niños les enseñan a "leer" en el marco de sus variedades vernáculas - en el sentido de las prácticas lingüísticas propias de su socialización primaria-, pero no les enseñan el aspecto discursivo de la literacidad académica. Y es que lo que es difícil en la escuela no es aprender a leer y a escribir, sino aprender a leer y escribir "de ciertas formas" asociadas a áreas de contenido académico y atadas a variedades de lenguaje especializado que resultan complejas, técnicas y alienantes para muchos aprendices.

Así, entonces, a pesar de que los alumnos requieren adoptar las convenciones del lenguaje escolar (y de la escritura escolar específicamente) no solo en el nivel de la palabra, sino también de la oración y del discurso, los profesores suelen enfocarse en los contenidos de los textos en desmedro de los patrones del lenguaje que construyen estos contenidos. Muchas veces las consignas de los maestros se reducen a la caligrafía, la ortografía, la puntuación y quizás el léxico, pero no abordan otros aspectos más de índole discursiva y epistémica. 
Un texto académico también involucra tomar en cuenta cómo presentar las ideas (a partir de nominalizaciones que condensan mayor información en una sola palabra), la manera como el escritor toma posición respecto de lo dicho (usando la tercera persona para tomar cierta distancia de lo declarado) y las estrategias de estructuración organizacional (utilizando una serie de conectores para combinar las cláusulas y lograr cohesión textual). Ahora bien, además de no explicitar las convenciones de la literacidad académica, los profesores tampoco suelen plantear claramente sus expectativas en relación con la producción lingüística de los estudiantes, aspecto que genera mucha confusión en ellos. Diversos autores han mostrado su preocupación ante este fenómeno. Christie (1985), por ejemplo, ha planteado que el lenguaje constituye el "currículo oculto" de la escolarización, en el sentido de que no se suele visibilizar la forma en que contribuye a reproducir la desigualdad; mientras que Lillis (2003) hace referencia a la enseñanza de la escritura académica como parte de una "práctica institucional del misterio".

Desde la perspectiva de la lingüística sociocultural, estas formas académicas asociadas a la escuela (como cualquier uso de los recursos lingüísticos) se conciben como sistemas simbólicos enraizados en la práctica social — que no pueden desligarse de valores sociales y culturales- y no como habilidades descontextualizadas y neutrales para la codificación y descodificación de símbolos gráficos. Esto significa que el lenguaje académico no constituye solo una técnica que puede ser aprendida en un curso introductorio sobre el tema. Más aún, no es suficiente que el alumno aprenda una serie de reglas sobre cómo este tipo de lenguaje incluye recursos léxicos, gramaticales y discursivos que son diferentes a los que encontramos en la interacción oral más informal. Y es que la literacidad académica - conceptualizada como maneras de usar los recursos lingüísticos - siempre se desarrolla en el marco de aprendizajes culturales y se adquiere como parte de la identidad de la gente (Gee, 2005).
En efecto, el lenguaje siempre es parte de la práctica social e involucra mucho más que el aspecto lingüístico. La noción de "práctica" es central para los estudios socioculturales de literacidad, pero también para una teoría del aprendizaje situado, que abarca mucho más que el uso del habla y de la escritura. La teoría de la práctica social (Lave, 1996) ha influido en el desarrollo de una noción de aprendizaje como participación en comunidades de práctica y, por lo tanto, como un aspecto integral e inseparable de la práctica social (Lave y Wenger, 1991). Todo esto significa que en cualquier proceso de aprendizaje, como parte de actividades situadas, las personas no solo adquieren habilidades, sino que participan como actores en el mundo y desarrollan identidades que los hacen miembros de comunidades específicas. Por lo tanto, cuando los alumnos aprenden la literacidad académica como parte de actividades particulares, no solo está en juego el desarrollo de habilidades, sino también la construcción de relaciones sociales con los otros miembros de la comunidad, el despliegue de un tipo de identidad y la membresía en la colectividad "académica", y la reproducción de los valores, intereses y compromisos implícitos de esta cultura en particular. Como lo señala Ivanič (1998), muchos estudiantes conciben esta literacidad académica como una especie de "juego" en el que se les pide que asuman una identidad que "no soy yo" y que no refleja la imagen que tienen de sí mismos. Por lo tanto, los conflictos y malentendidos que emergen entre estudiantes y profesores en relación con el tema de la literacidad académica no se restringen simplemente a la técnica de la escritura, las habilidades o la gramática, sino a aspectos que están relacionados con la identidad y la epistemología (Zavala, 2009).

Pero el tema de la literacidad académica no solo se vincula con la identidad y la epistemología, sino también con el poder y, en el caso que nos ocupa, con la justicia sociolingüística. Dentro de la mirada antes expuesta, la literacidad escolar no es neutral, sino que constituye una práctica lingüística enmarcada en una práctica social que ha 
ganado legitimidad por razones ideológicas y relaciones de poder. Ahora bien, aunque aprender a usar recursos lingüísticos de formas que cumplen las expectativas de la escuela constituye un reto para todos los estudiantes, es particularmente difícil para aquellos que han tenido pocas oportunidades de exponerse al uso de este tipo de lenguaje fuera de ella. Por lo tanto, en la medida en que no se aplica un enfoque explícito para la enseñanza del lenguaje, la institución escolar continúa privilegiando solo a estudiantes de ciertos contextos sociales, no solo en el aspecto del aprendizaje, sino también de las evaluaciones y las promociones. Después de todo, el lenguaje sirve como un medio muchas veces inconciente para evaluar, clasificar y diferenciar a los estudiantes, y así seguir perpetuando desigualdades y brechas.

Para terminar esta sección, quisiera precisar que es importante reconocer la inherente variabilidad de estas formas del lenguaje normalizadas y el hecho de que también se transforman. Si reconocemos que la literacidad constituye un proceso dinámico e interactivo de producción de significado socialmente situado y que los géneros son "recursos socialmente disponibles” (Fairclough, 1992), que no remiten a formas fijas de llevar a cabo eventos sociales dados, sino que pueden utilizarse de formas complejas y creativas (Martínez, 2005), hay que rechazar nociones estáticas e idealizadas del lenguaje y promover que los estudiantes experimenten con textos fluidos que trasciendan los géneros convencionales y los límites entre lenguas. No se trata entonces de imponer normas y reglas de textualidad, sino de permitir a los estudiantes que encuentren una voz en el discurso académico, que concilien sus diferentes identidades y que se inserten en esta comunidad con disposición y sintiéndose partícipes de ella.

\section{Reflexiones finales}

A lo largo de este artículo he tratado de hacer un contrapunto entre las prioridades de la educación contemporánea - desde una mirada específica del Perú- y la noción de justicia sociolingüística, en el sentido de una educación que busca empoderar a los estudiantes minorizados en relación con temas del lenguaje. En esta era del número - al decir de Ball (2008) - generalmente nos olvidamos lo que los números representan, valoramos solo lo que puede ser medible, no medimos aquello que es valioso y además no nos damos cuenta de que hay muchos aspectos que no pueden ser medibles pero que sí son valiosos.

La noción de justicia sociolingüística requiere dejar de lado una perspectiva descriptiva del lenguaje para pasar a una perspectiva crítica (Ivanič y Moss, 2004). Una perspectiva descriptiva para abordar el lenguaje representa la variedad, lo apropiado y el registro como si fueran fijos y por lo tanto incuestionables, lo que sugiere que es natural y justo que la realidad sea de esa manera. Según Ivanič y Moss (2004), la interpretación educativa de esta perspectiva es normativa, pues se induce a los estudiantes no solo a que se acomoden a la norma (y que accedan a los registros de poder), sino también a que acepten las cosas como son, pues se asume como correcto e inevitable que ciertos tipos de lenguaje tengan más prestigio que otros. En contraste, una perspectiva crítica del lenguaje representa la variedad, lo apropiado y el registro como elementos abiertos al cuestionamiento y al cambio. Los lingüistas y educadores críticos son explícitos acerca de la diferencia de estatus entre los diferentes tipos de lenguaje y cuestionan por qué prácticas distintas se consideran apropiadas en contextos diferentes. Más aún, los lingüistas y educadores críticos creen que el lenguaje está en constante flujo: los registros pueden mezclarse, y las reglas sobre lo apropiado pueden ser cuestionadas y eventualmente cambiadas (Fairclough, 1995). Precisamente por esta razón no podemos quedarnos en una valoración celebratoria de las formas lingüísticas de los estudiantes o en brindar las oportunidades para que estos accedan a las formas de poder. La perspectiva crítica implica además legitimar las formas que los estudiantes traen a la escuela y que son distintas a las que privilegia esta institución. 
Solo con una perspectiva crítica podemos reconocer que las prácticas del lenguaje generalmente valoradas en la escuela no son accesibles de forma igual para todos y que para algunos resulta más difícil acceder a ellas. De esta manera, cuando a algunos estudiantes les va menos bien que a otros, ya no se asume que esto es producto de la responsabilidad de los individuos, sino de los inflexibles (y arbitrarios) valores y normas de las instituciones educativas. Visibilizar el ejercicio del poder en el tema del lenguaje nos permite deconstruir el significante de la "igualdad de oportunidades" y darnos cuenta precisamente de que, a pesar del esfuerzo individual, no todos están en las mismas condiciones y tienen las mismas oportunidades para acceder a las recompensas sociales que el sistema educativo promete.

Con justa razón, Ladson-Billings (2006) rechaza el uso del término "brecha de rendimiento" y propone en su lugar el de "deuda educativa”, argumentando que no se trata solo de un cambio de término que obedece a las disparidades en logros académicos. Lo que resulta central aquí son las causas que se encuentran en la raíz de las disparidades y el sentido de la responsabilidad social que acompaña a esas causas. El discurso en torno a las brechas sugiere que cada individuo es responsable de su propia circunstancia educativa y que ciertos tipos de estudiantes deben "nivelarse" en relación con los otros, sin reconocer las formas en que la nivelación es muchas veces muy difícil debido a las barreras estructurales que la sociedad les ha impuesto. El lenguaje es precisamente un elemento central en estas barreras estructurales y ya es tiempo de que lo tomemos más en serio y en su real dimensión.

\section{Referencias}

Amrein, A. L. y Berliner, D. C. (2002). An analysis of some unintended and negative consequences of high-stakes testing. Tempe, AZ: Educational Policy Studies Laboratory, Arizona State University.

Au, W. (2011). Teaching under the new Taylorism: highstakes testing and the standardization of the $21^{\text {st }}$ century curriculum. Journal of Curriculum Studies, 43(1), 25-45.

Augoustinos, M., Tuffin, K., y Every, D. (2005). New racism, meritocracy and individualism: constraining affirmative action in education. Discourse \& Society, 16(3), 315-340.

Bale, J. (2010). International comparative perspectives on heritage language education policy research. Annual Review of Applied Linguistics, 30, 42-65.

Ball, S. (2008). The education debate. Londres: Policy Press.

Bernárdez, E. (2004). ¿Qué son las lenguas? (2. ${ }^{a}$ Ed.) Madrid: Alianza.

Blommaert, J., Collins, J. y Slembrouck, S. (2005). Spaces of multilingualism. Language \& Communication, 25, 197-216.

Blommaert, J. y Backus, A. (2011). Repertoires revisited: 'Knowing language' in superdiversity. Working papers in urban languages and literacies (vol. 67). Londres: King's College.

Bucholtz, M., López, A., Mojarro, A., Skapoulli, E., VanderStouwe, C. y Warner-García, S. (2014). Sociolinguistic justice in the schools. Student researchers as linguistic experts. Language and Linguistics Compass 8/4, 144-157.

Cameron, D. (1995). Verbal hygiene. Londres: Routledge.

Canagarajah, S. (1997). Safe houses in the contact zone: coping strategies of African American students in the academy. College Composition and Communication, 48(2), 173-196.

Christie, F. (1985). Language and schooling. En S. Tchudi (Ed.), Language, schooling and society (pp. 21-40). Upper Montclair, NJ: Boynton/Cook.

Clark, R. e Ivanič, R. (1997). The politics of writing. Londres: Routledge.

Cuenca, R. (2012). Sobre justicia social y su relación con la educación en tiempos de desigualdad. Revista Internacional de Educación para la Justicia Social, 1(1), 79-93.

Cummins, J. (2007). Pedagogies for the poor? Realigning reading instruction for low-income students with scientifically based reading research. Educational Researcher, 36(9), 564-572.

De León, L. (Ed.). (2010). Socialización, lenguajes y culturas infantiles: estudios interdisciplinarios. México D. F.: CIESAS.

De los Heros, S. (2012). Utopia y realidad: nociones sobre el estándar lingüistico en la esfera intelectual y educativa 
peruana. Madrid/Frankfurt/Lima: Iberoamericana/ Vervuert/IEP.

Fairclough, N. (1992). Discourse and social change. Londres: Polity Press.

Fairclough, N. (1995). Critical language awareness. Londres: Longman.

Flores, N. y Rosa, J. (2015). Undoing appropriateness: raciolinguistic ideologies and language diversity in education. Harvard Educational Review, 85(2), 149-171.

Fraser, N. (2005). Reframing justice in a globalizing world. New Left Review, 36, 69-88.

Freire, P. (1990). La naturaleza política de la educación: cultura, poder y liberación. Barcelona: Paidós.

Gazzola, M.; Templin, T. y Wickström, B. (Eds.). (2018). Language policy and linguistic justice. Economic, philosophical and sociolinguistic approaches. Suiza: Springer.

Gee, J. P. (2005). A strange fact about not learning to read. Situated language and learning. A critique to traditional schooling (pp. 7-20). Nueva York: Routledge.

Heath, S. B. (2004). El valor de la lectura de cuentos infantiles a la hora de dormir: habilidades narrativas en el hogar y en la escuela. En V. Zavala, M. Niño-Murcia y P. Ames (Eds.), Escritura y sociedad. Nuevas perspectivas teóricas y etnográficas (pp. 143-180). Lima: Red para el Desarrollo de las Ciencias Sociales en el Perú.

Irvine, J. (1989). When talk isn't cheap: Language and political economy. American Ethnologist, 16(2), 248-267.

Ivanič, R. (1998). Writing and Identity: The discoursal construction of identity in academic writing. Amsterdam: John Benjamins Publishing.

Ivanič, R. y Moss, W. (2004). La incorporación de las prácticas de escritura de la comunidad en la educación. En V. Zavala, M. Niño-Murcia y P. Ames (Eds.), Escritura y sociedad. Nuevas perspectivas teóricas y etnográficas (pp. 211-246). Lima: Red para el Desarrollo de las Ciencias Sociales en el Perú.

Kress, G. (2001). From Saussure to critical sociolinguistics. The turn towards a social view of language. En M. Wetherell, S. Taylor y S. Yates (Eds.), Discourse theory and practice: A reader (pp. 29-38). Londres: Sage y Open University.

Kroskrity, P. V. (2000). Regimenting languages: Language ideological perspectives. En Regimes of language: Ideologies, polities, and identities (pp. 1-34). Santa
Fe, NM: School of American Research Advanced Seminar Series.

Ladson-Billings, G. (2006). From the achievement gap to the education debt: Understanding achievement in U. S. schools. Educational Researcher, 35, 3-12.

Lamas Zoeger, L. (2015). La universidad privada en la construcción de subjetividades emprendedoras. Revista Peruana de Investigación Educativa, 7.

Lave, J. (1996). Teaching, as learning, in practice. Mind, Culture, and Activity, 3(3), 149-164.

Lave, J. y Wenger, E. (1991). Situated learning. Legitimate peripheral participation. Cambridge: Cambridge University Press.

Leeman, J. (2005). Engaging critical pedagogy: Spanish for native speakers. Foreign Language Annals, 38(1), 35-45.

Leeman, J. y King, K. (2013). Heritage language education: Minority language speakers, second language instruction, and monolingual schooling. En M. Bigelow y J. Ennser-Kananen (Eds.), Handbook of educational linguistics (pp. 210-223). Londres: Routledge.

Lemke, J. (1989). Semantics and social values. Word, 40(12), 37-50.

Lillis, T. (2003). Student writing as 'academic literacies': Drawing on Bakhtin to move from critique to design. Language and Education, 17(3), 192-207.

Luke, A.; Green, J. y Kelly, G. (2010). Introduction. What counts as evidence and equity? Review of Research in Education, 34, vii-xvi.

Madaus, G. F. y Clarke, M. (2001). The adverse impact of high-stakes testing on minority students: evidence from one hundred years of test data. En G. Orfeld y M. L. Kornhaber (Eds.), Raising standards or raising barriers? Inequality and high-stakes testing in public education (pp. 85-106). Nueva York: Century Foundation Press.

Martínez, G. A. (2005). Genres and genre chains: Post-process perspectives on heritage language writing in a South Texas setting. Southwest Journal of Linguistics, 24(1-2), 79-91.

Marx, K. y Engels, F. (1968). La ideología alemana. Montevideo: Pueblos Unidos.

Menken, K. (2010). NCLB and English language learners: Challenges and consequences. Theory into Practice, 49, 121-128. 
Michaels, S. (1986). Presentaciones narrativas: una preparación oral para la alfabetización con alumnos de primer curso. En La construcción social de la alfabetización (pp. 109-135). Barcelona: Paidós.

Milroy, J. (2007). The ideology of the standard language. En C. Llamas, L. Mullany y P. Stockwell (Eds.), Routledge companion to Sociolinguistics (pp. 133-139). Londres: Routledge.

Oliart, P. (2011). Discursos, tecnologías y prácticas de una reforma importada. Politicas educativas y la cultura del sistema escolar en el Perú. Lima: IEP/Tarea.

Pennycook, A. (2001). Critical applied linguistics. A critical introduction. Nueva Jersey: Lawrence Erlbaum.

Philips, Susan (1983). The invisible culture: Communication in classroom and community on the Warm Springs Indian reservation. Nueva York: Longman.

Piller, I. (2016). Linguistic diversity and social justice. An introduction to applied sociolinguistics. Oxford: Oxford University Press.

Rosa, J., y Burdick, C. (2017). Language ideologies. En O. García, N. Flores, y M. Spotti (Eds.), The Oxford handbook of language and society (pp. 103-123). Oxford: Oxford University Press.

Ruiz Bravo, P.; Rosales, J. L. y Neira, E. (2006). Educación y cultura: la importancia de los saberes previos en los procesos de enseñanza-aprendizaje. En M. Benavides (Ed.), Los desafios de la escolaridad en el Perú (pp. 79-156). Lima: GRADE.

Rymes, B. (2014). Communicating beyond language. Everyday encounters with diversity. Nueva York: Routledge.

Schleppegrell, M. (2005). The language of schooling. A functional linguistics perspective. Londres: Routledge.

Scollon, R. y Wong Scollon, S. (1981). Narrative, literacy and face in interethnic communication. Nueva Jersey: Ablex.

Siegel, J. (2006). Language ideologies and the education of speakers of marginalized language varieties: Adopting a critical awareness approach. Linguistics and Education, 17, 157-174.

Snow, C. E., y Uccelli, P. (2009). The challenge of academic language. En D. R. Olson y N. Torrance (Eds.),
The Cambridge handbook of literacy (pp. 112-133). Cambridge: Cambridge University Press.

Street, B. (1996). Literacy and power? Open Letter, 6(2), 7-16. Sídney, Australia: UTS.

Torres, J. (2001). Educación en tiempos de neoliberalismo. Madrid: Morata.

Van Deusen-Scholl, N. (2003). Toward a definition of heritage language: sociopolitical and pedagogical considerations. Journal of Language, Identity, and Education, 2(3), 211-230.

Velasco, P. y García, O. (2014). Translanguaging and the writing of bilingual learners. The Bilingual Research Journal, 37(1), 6-23.

Woolard, K. (1998). Language ideology as a field of inquiry. En B. Schieffelin, K. Woolard y P. Kroskrity (Eds.), Language ideologies. Practice and theory (pp. 3-47). Oxford: Oxford University Press.

Zavala, V. (2009). ¿Quién está diciendo eso?: literacidad académica, identidad y poder en la educación superior. En J. Kalman y B. Street (Eds.), Lectura, escritura y matemáticas como prácticas sociales: Diálogos con América Latina (pp. 348-363). México D. F.: Siglo XXI Y CREFAL.

Zavala, V. (2012). Dilemas ideológicos en torno a la educación intercultural bilingüe: el caso de la lectura en quechua. Revista de la Sociedad de Investigación Educativa Peruana, 4, 77-104.

Zavala, V. (2015). 'It will emerge if they grow fond of it': Translanguaging and power in Quechua teaching. Linguistics and Education, 32, 16-26.

Zavala, V.; Niño-Murcia, M. y Ames, P. (2004). Escritura y sociedad: nuevas perspectivas teóricas y etnográficas. Lima: Red para el Desarrollo de las Ciencias Sociales en el Perú.

Zavala, V. y Córdova, G. (2010). Decir y callar. Lenguaje, equidad y poder en la universidad peruana. Lima: Fondo Editorial de la PUCP.

Zavala, V.; Mujica, L.; Córdova, G. y Ardito, W. (2014). Qichwasimirayku: Batallas por el quechua. Lima: Fondo Editorial de la PUCP.

Žižek, S. (1997). Multiculturalism or the cultural logic of multinational capitalism. New Left Review, 1(225), 28-51.

How to reference this article: Zavala, V. (2019). Justicia sociolingüística para los tiempos de hoy. Íkala, Revista de Lenguaje y Cultura, 24(2), 343-359. Dor: 10.17533/udea.ikala.v24n02a09 